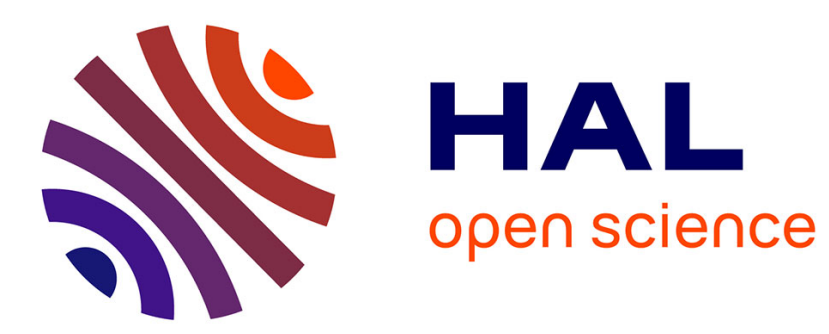

\title{
Analytical description of the topological interaction between magnetic domain walls in nanowires
}

\author{
A. Pivano, V.O. Dolocan
}

\section{To cite this version:}

A. Pivano, V.O. Dolocan. Analytical description of the topological interaction between magnetic domain walls in nanowires. Physical Review B, 2020, 101 (1), 10.1103/PhysRevB.101.014438 . hal03153333

\section{HAL Id: hal-03153333 \\ https://hal.science/hal-03153333}

Submitted on 26 Feb 2021

HAL is a multi-disciplinary open access archive for the deposit and dissemination of scientific research documents, whether they are published or not. The documents may come from teaching and research institutions in France or abroad, or from public or private research centers.
L'archive ouverte pluridisciplinaire HAL, est destinée au dépôt et à la diffusion de documents scientifiques de niveau recherche, publiés ou non, émanant des établissements d'enseignement et de recherche français ou étrangers, des laboratoires publics ou privés. 


\title{
Analytical description of the topological interaction between magnetic domain walls in nanowires
}

\author{
A. Pivano ${ }^{1, *}$ and V.O. Dolocan ${ }^{1, \dagger}$ \\ ${ }^{1}$ Aix-Marseille Université, CNRS, IM2NP UMR7334, F-13397 Marseille Cedex 20, France
}

(Dated: January 14, 2020)

\begin{abstract}
Magnetic domain walls in nanowires behave as particles interacting through the exchange field. As topological objects, their interaction is determined by their chirality or topological charge. We investigate analytically the topological repulsion between magnetic domain walls with same topological charge in nanostripes (with easy-plane magnetization) and show that it decays algebraically, as $r^{-2}$, being part of a larger class of interactions that produce topological long-range order in two dimensions. We compare the topological repulsion between the walls with other type of fundamental interactions with exponential spatial decay, like the Yukawa-Reid potential, and with micromagnetic simulations. We determine that trains of such walls can be well described analytically and can be displaced regularly in nanowires leading to practical applications.
\end{abstract}

PACS numbers: $75.60 . \mathrm{Ch}, 75.10 . \mathrm{Hk}, 75.40 . \mathrm{Mg}$

\section{INTRODUCTION}

The interaction forces of nature are few and theirs exact spatial variation is difficult to determine from first principles. In quantum field theory, the fundamental interactions are mediated by massless spin one particles like gluons for the strong interaction or photons for the electromagnetic interaction. In the non-relativistic case, these interactions are described by an interaction potential. In practice, some phenomenological model is often employed as in the case of nucleon-nucleon interaction where a Reid-type (Yukawa) potential is frequently used and compared with experimental results[1]. The Yukawa-type potential is equally used to describe the inter-particle interaction in strongly coupled systems like ultracooled neutral plasmas[2] as well as in colloidal suspensions[3] (the so-called Yukawa systems).

In several condensed matter areas and in field theories, the more localized excitations of nonlinear systems are considered as quasiparticles and described within the collective-variable approach[4]. The interactions between these quasiparticles, like vortices in superconductors, which have an equivalent in cosmology (global strings) $[5,6]$, decay monotonically as $\mathrm{r}^{-2}$ in thin superconducting films or exponentially in the bulk[7]. The exponential repulsive interaction is also found in more mundane interactions as the one between two pedestrians[8].

In magnetic systems, the interaction potential is dominated at short range by the exchange interaction. The exchange interaction is model dependent, the most common model being based on the Heisenberg Hamiltonian, from which is derived the semi-classical exchange interaction proportional to the square of the magnetization gradient. This Heisenberg derived dependence is

\footnotetext{
* Current address: CEA, DEN, DTN/SMTA/LEAG, Cadarache F-13108 Saint Paul-lez-Durance, France

† voicu.dolocan@im2np.fr
}

heavily used in numerical calculations of ferromagnets (micromagnetics) [9].

Domain walls (DW) confined in magnets at the nanoscale can be considered as particles (macrospins) which interact through the exchange field. The DWs in the confined structures are transverse or vortex DWs depending on the samples dimensions[10]. The DWs are formed from two or more elementary topological defects with an integer winding number like vortices in the bulk or fractional winding number which are half-vortices confined to the edges $[11,12]$. In a planar nanowire with inplane magnetization (nanostrip), the chirality of the DW is protected by topology and is also called a topological charge[13]. A pair of in-plane DWs with opposite topological charges (opposite fractional edge defects) can be created or annihilated spontaneously, but a pair of DWs with the same topological charge form a stable magnetic texture - a soliton-soliton pair[14] (due to the "topological repulsion", see Fig. 1(a) where four transverse DWs with same topological charge are pinned in a nanostrip). In a $3 \mathrm{D}$ cylindrical nanowire, it was shown that a pair of DWs with the same initial topological charge form a metastable state that anihilates after a finite time, due to the relative rotation of the walls[15] and the non conservation of the total topological charge. The injection of DWs in a nanowire with reliable chirality control has been demonstrated experimentally[16]. The total topological charge is conserved during DW interaction and a train of this type of DWs can be displaced jointly in the nanostrip by a polarized current leading to practical applications[17, 18].

The interaction potential beteen the DWs can also be viewed as mediated by the topological defects. The interaction between vortex DWs was studied analytically based on the Thiele's approach in a $2 \mathrm{D}$ anisotropic Heisenberg model[19, 20] and also experimentally[21]. In the majority of cases only the dynamics of one DW is studied and simulated micromagnetically or the dynamics of well separated DWs (different nanowires or nanolayers) that interact through the dipolar field 
[22-31]. In few cases, the DWs interaction in the same nanowire was studied experimentally and micromagnetically[32, 33]. Analytically, the interaction potential between transverse DWs in nanostripes was considered only based on a the multipole expansion[34], which was tested numerically for DWs pinned at artificial constrictions situated at large distances[15, 29, 35] where the dipolar interaction dominates. To be able to calculate analytically the dynamics of a train of transverse DWs in a nanowire, a pertinent model should take into account the repulsive (topological) interaction which is important at short range.

In this paper we address this issue, establishing the transverse DW interaction potential using trial models. We test our phenomenological model numerically on the fast-dynamics of two, three and four transverse DWs initially pinned at symmetric notches along a nanostrip, and submitted to ultrashort current pulses. Our analysis is similar to the classical model of a $1 \mathrm{D}$ chain of interacting particles. In our model, we take into account only nearest neighbor DW exchange interaction and the dipolar interactions up to the third neighbor. We determine that a power-law spatial variation of the DW exchange interaction, of $r^{-2}$ type, gives quantitatively good results when compared to the micromagnetic calculations, similar with the Heisenberg exchange and the interaction of superconducting vortices in thin films. In a 2D XY model, the interaction between charged particles (vortices) was shown to decrease logarithmically rather than exponentially below a topological phase transition[36]. We compare the obtained power-law behavior with an exponential or a Yukawa potential and discuss the observed differences in the DWs dynamics and depinning curents. We also determine that the transient effects related with the DW inertia-like behavior[37-41], due to deformation of the DW, diminish when the interaction between several DWs is considered as symmetric interactions on both sides annihilate the deformations of the walls. Our study shows that a simple analytical model gives good quantitative results even when the interaction between several DWs is considered, paving the way for calculating phase diagrams in larger memory racetracks.

This article is organized as follows. In Sec. II, we present the stochastic 1D model used to calculate the interaction between DWs. In Sec. III, we compute and investigate the phase diagram of the DW dynamics in an infinite nanostrip at $\mathrm{T}=0 \mathrm{~K}$ and at room temperature. Concluding remarks are presented in Sec. IV.

\section{MODEL}

To determine the interaction potential between transverse magnetic DWs, it's necessary to control the position and the topological charge of the DWs. In the following, the position is controlled by pinnig at notches and the topological charge is fixed initially as the injection with chirality control has already been proven experimentally[16]. The demagnetizing energy keeps the topological charge fixed for the DWs up to reasonable high external applied current. We consider several pinned transverse walls with the same topological charge in an infinite Ni nanostrip (saturation magnetization $\mathrm{M}_{s}=477 \mathrm{kA} / \mathrm{m}$, exchange stiffness parameter $\mathrm{A}=$ $1.05 \times 10^{-11} \mathrm{~J} / \mathrm{m}$, spin polarization $\mathrm{P}=0.7$ ) with a cross section of $\mathrm{L}_{y} \times \mathrm{L}_{z}=60 \times 5 \mathrm{~nm}^{2}$. No magnetocrystalline anisotropy is considered, the shape anisotropy insures that the easy axis is in-plane. The strip has rectangular symmetric double notches, with dimension $20 \times 9$ $\times 5 \mathrm{~nm}^{3}$, and separated by $80 \mathrm{~nm}$. Fig. 1(a) shows the equilibrium position of a train of four neighboring (situated in neighboring notches at $80 \mathrm{~nm}$ distance) transverse DWs: two pairs of head-to-head (HH) DWs and tail-totail (TT) DWs of same chirality (and inverse polarity at theirs centers) to ensure the topological stability and repulsion between them. Each DW sits in a potential well created by the notches[42, 43]. The form of the pinning potential was determined from micromagnetic simulations and is presented elsewhere[44] (harmonic at the notches and sinusoidal between them).

The DWs are displaced simultaneously by a series of periodic spin polarized current pulses applied along the stripe long axis ( $x$-direction). The geometry of the current pulse is described in Fig. 1(b): $\mathrm{t}_{r}, \mathrm{t}_{s}, \mathrm{t}_{f}$ and $\mathrm{t}_{z}$ are the rise, stable, fall time and zero-current time respectively. The nonadiabatic parameter is set to $\beta=2 \alpha$, if not specified otherwise.

The DW dynamics was computed using the onedimensional DW model[22, 45] considering the DWs interaction and by 3D micromagnetic simulations with the MUMAX3 package [46]. In both cases, the magnetization dynamics is determined from the Landau-LifschitzGilbert (LLG) equation with adiabatic and non-adiabatic spin-transfer torques[47]:

$\dot{\mathbf{m}}=-\gamma_{0} \mathbf{m} \times \mathbf{H}_{e f f}+\alpha(\mathbf{m} \times \dot{\mathbf{m}})-(\mathbf{u} \cdot \nabla) \mathbf{m}+\beta \mathbf{m} \times(\mathbf{u} \cdot \nabla) \mathbf{m}$

where $\mathbf{m}$ is the normalized magnetization, $\gamma_{0}$ is the gyromagnetic ratio, $\mathbf{u}=\mathbf{j}_{e} P \mu_{B} / e M_{s}$ is the spin drift velocity, $P$ the spin polarization of conductions electrons, $\mu_{B}$ the Bohr magneton and $\mathbf{j}_{e}$ the applied current density. No additional exotic torques (like the ones due to the spinHall or Rashba effect) were considered. The temperature is considered in the LLG equation as a thermal field added to the effective field. The thermal field has zero average and is uncorrelated in time and space and its magnitude is the same as the Gaussian noise introduced in the $1 \mathrm{D}$ model below.

The analytical equations of motion used are based on the 1D model of the DW (collective coordinates: average DW center position $X$ and azimuthal angle $\psi$ ) [48, 49]: 


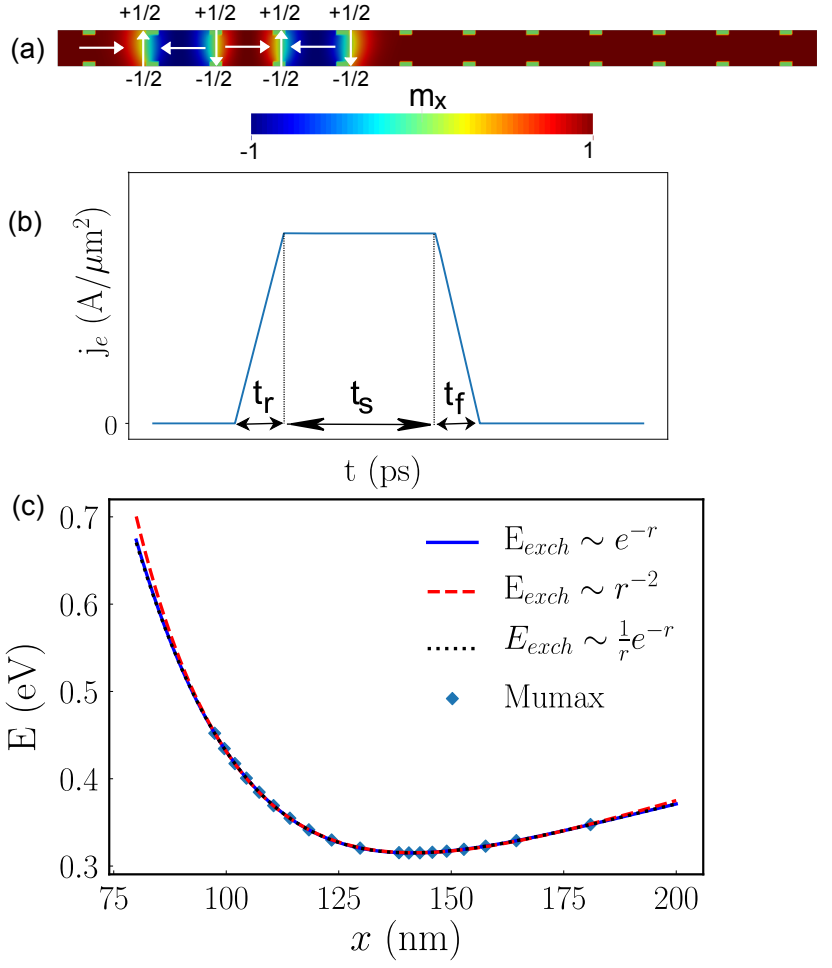

FIG. 1: (Color online) (a) Simulated structure with four magnetic domain walls with same topological

charge are pinned at symmetric notches: two head-to-head and two tail-to-tail DWs. The fractional winding numbers of the edge defects are indicated for each DW. (b) Current pulse shape used to displace the domain walls.(c) Interaction energy of two domain walls in a nanowire without notches (symbols) as determined from micromagnetic simulations. The curves represent modeling with different trial functions.

$$
\begin{aligned}
\left(1+\alpha^{2}\right) \dot{X}= & -\frac{\alpha \gamma \Delta}{2 \mu_{0} M_{s} S} \frac{\partial E}{\partial X}+\frac{\gamma \Delta}{2} H_{k} \sin 2 \psi \\
& +q p \frac{\gamma}{2 \mu_{0} M_{s} S} \frac{\partial E}{\partial \psi}+(1+\alpha \beta) u+\eta_{\psi}-\alpha \eta_{X} \\
\left(1+\alpha^{2}\right) \dot{\psi}= & -q p \frac{\gamma}{2 \mu_{0} M_{s} S} \frac{\partial E}{\partial X}-\frac{\gamma \alpha}{2} H_{k} \sin 2 \psi \\
& -\frac{\alpha \gamma}{2 \Delta \mu_{0} M_{s} S} \frac{\partial E}{\partial \psi}+q p \frac{\beta-\alpha}{\Delta} u+\eta_{X}+\alpha \eta_{\psi}
\end{aligned}
$$

with $\Delta(t)=\Delta[\Psi(t)]=\pi \sqrt{\frac{2 A}{\mu_{0} M_{S}{ }^{2} \sin ^{2} \psi+\mu_{0} M_{S} H_{k}}}$ the DW width, $H_{k}$ the DW demagnetizing field, $\eta_{X}$ and $\eta_{\psi}$ represent stochastic Gaussian noise with zero mean value and correlations $\left\langle\eta_{i}(t) \eta_{j}\left(t^{\prime}\right)\right\rangle=\left(2 \alpha k_{B} T\right) /\left(\mu_{0} M_{s} \Delta S\right) \delta_{i j} \delta(t-$ $\left.t^{\prime}\right)$. $E$ is the potential energy of the DW that includes the internal energy, the interaction energy with other DWs and the pinning energy. The azimuthal angle of the DW $\psi$ represents the conjugate momentum in the Lagrangian formulation. The interaction energy between DWs separated by $r_{i j}$ was modeled as $E_{i n t}=E_{\text {exch }}+E_{m m}+E_{d d}$, where

$$
\begin{aligned}
& E_{m m}^{i j}=\frac{a_{2} D_{2}}{r_{i j}} Q_{i} Q_{j}, E_{d d}^{i j}=a_{3}\left(\frac{D_{3}}{r_{i j}}\right)^{3} \cos \left(\psi_{i}-\psi_{j}\right) \\
& E_{\text {exch }}^{H}=a_{1}\left(\frac{D_{1}}{r_{i j}}\right)^{2}, E_{\text {exch }}^{\text {exp }}=a_{1} e^{-r_{i j} / D_{1}} \\
& E_{\text {exch }}^{Y}=\frac{a_{1} D_{1}}{r_{i j}} e^{-r_{i j} / D_{1}}
\end{aligned}
$$

represent the monopole-monopole $(\mathrm{mm})$, the dipoledipole $(d d)$ interaction and the DW exchange interaction ("topological repulsion"). The topological charge of the DW is $q=\frac{1}{\pi} \int \mathrm{d} x \partial_{x} \psi= \pm 1$ and is related with the direction of rotation of the in-plane magnetization when traversing the DW and $p= \pm 1$ represents the direction of the magnetization at the DW center along the $y$-axis (width). While both HHDW and TTDW can have a positive or negative topological charge and direction $p$, the product $Q=q \cdot p$ is always equal to +1 for a HHDW and to -1 for a TTDW. Therefore, the $m m$ and $d d$ interactions between nearest neighbors HHDW and TTDW of same topological charge but opposite $p$ directions are always negative, meaning attractive. We introduce a repulsion term, in the form of a "topological" or DW "exchange" interaction in a phenomenological manner as shown by the $E_{\text {exch }}$ terms of Eq. (3). Several trial functions were used and compared based on the asymptotic behavior of fundamental potentials, the interaction potential which correlates best with the micromagnetic simulations is the $r^{-2}$ decay. We only considered nearest neighbor DW exchange interaction, but $m m$ and $d d$ interaction was considered up to the third neighbor (see below the discussion on the displacement of the four DWs).

The parameters $a_{i}$ and $D_{i}$ were determined by comparing the obtained phase diagrams with the micromagnetic simulations. As the number of parameters is large, the starting values were chosen by fitting the micromagnetic results obtained for two DWs (a HHDW and a TTDW) initially situated at $80 \mathrm{~nm}$ distance in a very long nanowire of same section and without notches (Fig 1(c)). The two DWs repel each other at closer distance until around an equilibrium position of $140 \mathrm{~nm}$, beyond which the interaction becomes attractive due mainly to the long range dipolar interaction. These initial parameters were modified in the case of the pinned DWs as to follow closely the micromagnetic phase diagrams, but the order of magnitude was maintained.

For the micromagnetic computations, the strip was discretized into a mesh with a cell size of $2 \times 3 \times 2.5 \mathrm{~nm}^{3}$, inferior to the exchange length $(\sim 5 \mathrm{~nm})$. The DW dynamics 
is studied in an infinity long wire, where the magnetic charges at the ends of the nanostrip are compensated.

\section{RESULTS}

Our analysis of the DWs dynamical interaction begins with the study of the impact of the different interaction potential trial functions on the phase diagram obtained when a symmetric pulse (stable time $\mathrm{t}_{s}$ - current amplitude $\mathrm{j}_{e}$ ) or an asymmetric pulse (rise time $\mathrm{t}_{r}-\mathrm{j}_{e}$ ) are applied to the pinned DWs at $\mathrm{T}=0 \mathrm{~K}$. Afterwards, the particularities of the DWs motion at room temperature are discussed for the different interaction terms. The last subsection details the influence of the transient displacement on the DWs dynamics.

\section{A. Influence of the DW exchange energy on the phase diagrams at $T=0 K$}

To evaluate the impact of the different DW exchange terms on the DWs coupled dynamics, we computed $400 \times 300$ point-by-point analytical phase diagrams integrating the Eqs.(2) with a fourth order Runge-Kutta scheme. The phase diagrams represent the relative position of the train of DWs after periodic spin-polarized current pulses are applied to them. The current pulses are varied in length, amplitude or shape and the correlated displacement of the DWs is extracted after several periodic current pulses. When the DWs are displaced collectively keeping the same relative distance between them, we consider that an expected and desired state is realized. These collective regular displacements form bands depending on the pulse characteristics and the interaction potential between the DWs. The analytical diagrams are compared with the micromagnetic ones $(24 \times 31$ points). As previously determined[44], the range of the current amplitude was chosen $\left(\leq 10 \mathrm{~A} / \mu \mathrm{m}^{2}\right)$ as to to have only viscous motion (no precession) for the pulse duration used ( $\lesssim 1.5 \mathrm{~ns}$ ), which is on the same order of magnitude with access or reading/writing time in possible magnetic memories based on DWs. At high current amplitude or longer pulse duration, an antivortex appears when a DW depins from a notch[50, 51]. The antivortex will perturb the systematic motion of the DWs and their mutual interaction. In the results shown below, an antivortex appears only in a few points in the upper right quadrant of the micromagnetic phase diagrams (detailed in ref.44) where the symbols are missing, and does not influence our results.

Our analysis starts with a train of a HHDW and TTDW having the same topological charge and situated in neighboring pinning centers (separated by 80nm). The initial distance between the DWs ensures that their repulsive interaction is still important as determined from their equilibrium positions and Fig. 1(c). In Fig. 2 we present the results for various $\alpha$ and $\beta$ parame- ters (corresponding to the $\mathrm{Ni}$ values at $0 \mathrm{~K}$ and room temperature[52]) and several pulse shapes. In the upper panels, contour plots for the different bands are shown obtained with the 1D model, while the lower panels present a superposition of the 1D model diagrams (represented by colors) with the micromagnetic ones (symbols). The two DWs move together after a pulse application due to the spin transfer torque (STT) in the direction of the electron movement, but the final DW position can be in the opposite direction due to the transitory motion (automotion)[37-41]. We indexed the different regions in the phase diagram based on the relative position of the two DWs as follows: we call the state 0, when the DWs stayed in their initial notch (position) after the application of the pulse (pinned case), state +1 if the two DW went to the next notch in the direction of the electron flow (of the STT) keeping the same distance between them or state -1 if the two DW went to the next notch in the direction opposite of the electron flow. The higher number states were indexed in the same way $(+2$ means displacement of both DWs to the second next notch in STT direction). The state $\mathrm{u}$ is an unintended state (like depinning of one DW), where the DWs do not keep the initial relative distance between them. This state appear generally as a transition region between the other states. As our calculation is done on a finite sample of an infinite nanostrip, to be able to compare to the micromagnetic simulations, the number of bands is finite and the upper right region, that shows an unintended state, correspond to the DW reaching the nanowire (finite sample) end. The states were determined after the application of at least four periodic pulses that displace the DWs between their initial position and the desired position back and forth.

As observed in the lower panels of Fig. 2, the 1D model DW repulsive interaction varying in $r^{-2}$ (called DW Heisenberg-exchange) agrees quantitatively with the micromagnetic simulations up to the third band, afterwards a small shift appears. In the upper panels, the contour plots of only the first bands are shown for different repulsive interaction and different material and pulse parameters. In panel (a), for a symmetric pulse shape $\left(\mathrm{t}_{r}=\mathrm{t}_{f}=5 \mathrm{ps}\right)$ and $\alpha=0.02(\beta=2 \alpha)$, the contour plots obtained with the three-type of DW exchange interaction are superposed with the results obtained with no repulsive interaction (dash-dotted line). As can be observed, even in absence of the repulsive interaction, the two DWs can still be displaced synchronously due to the periodic pinning potential and the ultrashort pulses, but the depinning current increases to $3.05 \mathrm{~A} / \mu \mathrm{m}^{2}$ from 2.60 $\mathrm{A} / \mu \mathrm{m}^{2}$ above $\mathrm{t}_{s}=0.6 \mathrm{~ns}$, and the bands increase and are more deformed. This situation is equivalent with the case of two DWs initially separated by a larger distance than the range of the repulsive interaction[53]. The depinnig current diminishes when an exponential or Yukawa-type DW exchange is used to $2.21 \mathrm{~A} / \mu \mathrm{m}^{2}$ and $1.87 \mathrm{~A} / \mu \mathrm{m}^{2}$ respectively above $t_{s}=0.6 \mathrm{~ns}$. The variation of the repulsive interaction impacts slightly the shape and surface of the 

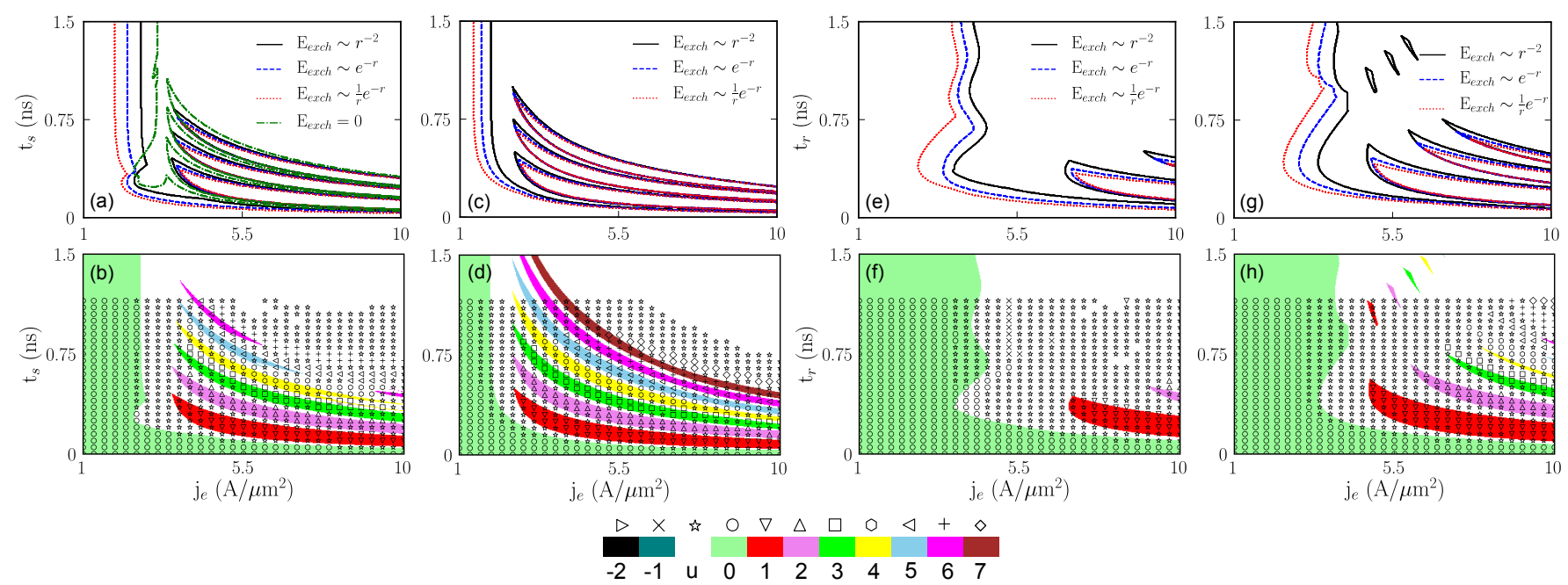

FIG. 2: (Color online). Contour plots of the different bands obtained for a train of two neighboring DWs with different type of DW exchange interaction at $\mathrm{T}=0 \mathrm{~K}$ using the $1 \mathrm{D}$ model are represented in the upper panels (a), (c),

(e) and (g). In the lower panels, the 1D results (colored regions) obtained for a Heisenberg type DW exchange interaction are compared with the micromagnetic calculations (scattered symbols) for the same pulse characteristics and $\alpha$ and $\beta$ parameters as the upper panels. The numbering of the bands is as follows: positive bands correspond to the DWs moving collectively in the direction of the electron flow with the same initial relative distance between

them, negative numbers to the DWs moving collectively in the opposing direction with the same initial relative

distance between them, zero state correspond to the DWs staying pinned at initial positions and 'u' to the unintended states in which the DWs do not move synchronously in either direction. The parameters used are: (a) and (b) $\mathrm{t}_{s}$ variable, $\mathrm{t}_{r}=\mathrm{t}_{f}=5 \mathrm{ps}, \alpha=0.02, \beta=0.04$, (c) and (d) $\mathrm{t}_{s}$ variable, $\mathrm{t}_{r}=\mathrm{t}_{f}=5 \mathrm{ps}, \alpha=0.05, \beta=0.1,(\mathrm{e})$ and (f) $\mathrm{t}_{r}$ variable, $\mathrm{t}_{s}=\mathrm{t}_{f}=5 \mathrm{ps}, \alpha=0.02, \beta=0.04,(\mathrm{~g})$ and $(\mathrm{h}) \mathrm{t}_{r}$ variable, $\mathrm{t}_{s}=\mathrm{t}_{f}=5 \mathrm{ps}, \alpha=0.05, \beta=0.1$.

upper bands, the most important change is on the depinning current for the symmetric pulse (panels (a) and (c)). For asymmetric pulses (panels (e) and (g)), where $\mathrm{t}_{s}=\mathrm{t}_{f}=5 \mathrm{ps}$ and the rise time $\mathrm{t}_{r}$ is varied, the change in form and surface of the bands is more important as compared to the symmetric pulses. Increasing the damping parameter $\alpha$ to 0.05 (with $\beta=2 \alpha$ ), as shown in panels (c) and $(\mathrm{g})$, shifts all the bands to lower currents, including the depinning value. We used in all the calculations the same parameters for the $m m$ and $d d$ interaction: $a_{2}=$ $0.2 \mathrm{eV}, a_{3}=0.02 \mathrm{eV}, D_{2}=D_{3}=500 \mathrm{~nm}$. For the different DW exchange interactions, the parameters used are: $a_{1}=1.2 \mathrm{eV}$ and $D_{1}=350 \mathrm{~nm}$ for $E_{\text {exch }}^{H}, a_{1}=20 \mathrm{eV}$ and $D_{1}=150 \mathrm{~nm}$ for $E_{\text {exch }}^{\text {exp }}$ and $a_{1}=90 \mathrm{eV}$ and $D_{1}=150 \mathrm{~nm}$ for $E_{\text {exch }}^{Y}$. These parameters were chosen to fit best the micromagnetic depinning line of shortest pulse length. In-depth details about the comparison between analytic and micromagnetic calculations are given in the Supplemental Material.

The importance of the pulse shape and length was inferred by decoupling the Eqs.(2) [54, 55] :

$$
\ddot{X}=-\frac{\dot{X}}{\tau_{d}}-\frac{1}{m} \frac{d E}{d X}+\frac{\beta}{\alpha \tau_{d}} u+\frac{1+\alpha \beta}{1+\alpha^{2}} \dot{u}
$$

with $m=\frac{2 \alpha S \mu_{0} M_{s} \tau_{d}}{\Delta \gamma_{0}}$ the DW mass and $\tau_{d}=\frac{1+\alpha^{2}}{\alpha \gamma_{0} H_{k}}$ the damping time of the wall in the pinning potential. Here, the damping time is $0.27 \mathrm{~ns}$ for $\alpha=0.05$ and $0.68 \mathrm{~ns}$ for $\alpha=0.02$, so the third term in Eq. (4) is more important for higher damping parameter $\alpha$, resulting in a lower depinning current as observed from Fig. 2(a) and (c) 1.85 $\mathrm{A} / \mu \mathrm{m}^{2}$ compared to $2.60 \mathrm{~A} / \mu \mathrm{m}^{2}$ for Heisenberg DW exchange). The depinning current increases to $3.68 \mathrm{~A} / \mu \mathrm{m}^{2}$ (lowest value) in panel (e) and $3.39 \mathrm{~A} / \mu \mathrm{m}^{2}$ in panel (g) for a longer rise time as the last term of Eq. (4) is directly proportional to the current derivative. The second term of Eq. (4) gives a hint to the different depinning current obtained for various DW exchange forms used.

\section{Case of four interacting DWs}

The influence of the repulsive interaction between nearest neighbors DWs was further studied by extending the analytical calculation up to four DWs of same topological charge. We describe here the case of a chain of four consecutive interacting DWs: we consider the topological repulsive interaction between first neighbors in the forms presented above, along with the monopole-monopole and dipole-dipole interaction between each pair of DWs. The $\mathrm{mm}$ and $d d$ interactions are attractive between first neighbors, repulsive between second neighbors and attractive between third neighbors. The parameters $a_{i}$ and $D_{i}$ were kept constant for first-, second- and thirdneighbor $m m$ and $d d$ interactions with the values given 


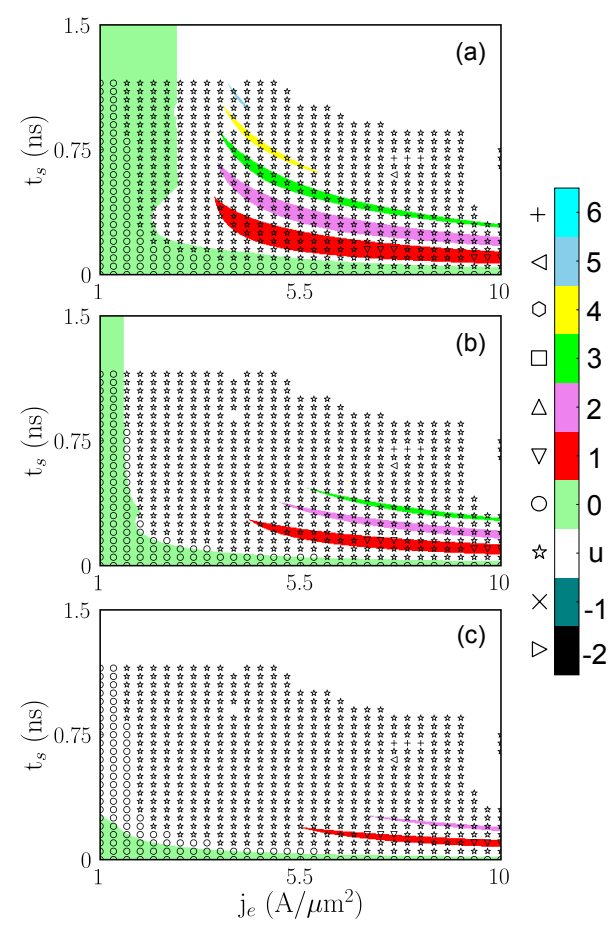

FIG. 3: (Color online) Influence of the Heisenberg DW exchange energy magnitude on the bands for a train of four neighboring DWs for $\alpha=0.02$ and $\beta=0.04$ : (a) $\mathrm{a}_{1}=$ $0 \mathrm{eV}$ (no DW exchange), (b) $\mathrm{a}_{1}=1.0 \mathrm{eV}$ and (c) $\mathrm{a}_{1}=$ $1.4 \mathrm{eV}$. The $1 \mathrm{D}$ results (colored regions) obtained for a Heisenberg type DW exchange interaction are compared with the micromagnetic calculations (scattered symbols) at $\mathrm{T}=0 \mathrm{~K}$. The pulse stable time was varied, with $\mathrm{t}_{r}=\mathrm{t}_{f}=5 \mathrm{ps}$ and $\mathrm{t}_{z}=10 \mathrm{~ns}$.

above.

Fig 3 displays the influence of the magnitude of the DW exchange interaction between nearest neighbors for $\alpha=0.02$ using a Heisenberg-type DW exchange. The difference between no DW exchange (panel (a)) and a DW exchange of the same order of magnitude as used for a train of $2 \mathrm{DWs}$ is much more drastic as the depinning current and the band width diminish strongly when the DW exchange is turned on (panel (b), $a_{1}=1.0 \mathrm{eV}$ ). A further increase of the DW exchange interaction will lead to the quasi-suppression of the depinnig current (displaced to lower values), but also of the bands (panel (c), $a_{1}=1.4 \mathrm{eV}$ ). The analytic results follow very well the micromagnetic ones for the depinning current line (panel (b)) and semi-quantitatively the band form, which validates the model.

To further investigate the consequences of the DW exchange interaction type on the DW dynamics, we present the evolution of the phase diagrams in the Fig. 4 for different pulse shape and damping parameter. The panels (a) and (b) show the contour plots of the first bands due to a symmetric current pulse shape $\left(\mathrm{t}_{r}=\mathrm{t}_{f}=5 \mathrm{ps}\right)$ and for $\alpha=0.02$ and 0.05 respectively, while the pan-
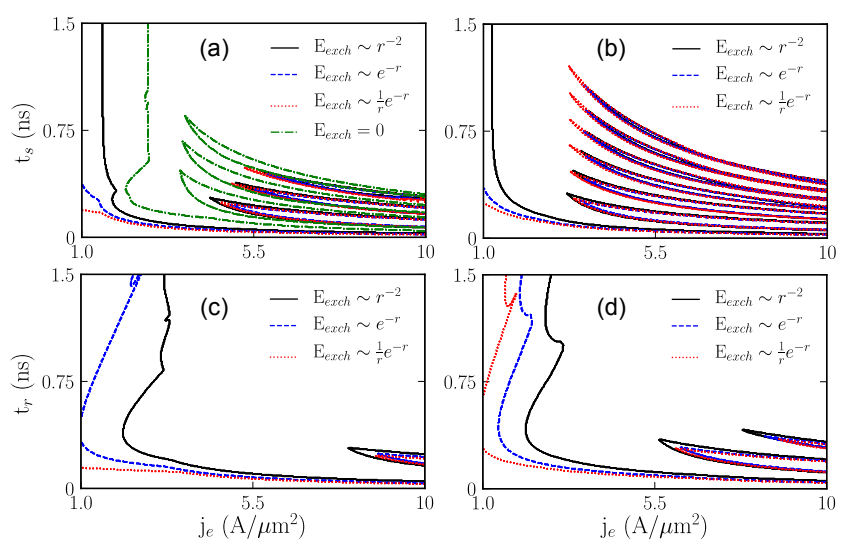

FIG. 4: (Color online) Contour plots of the different bands obtained for a train of four neighboring DWs

(separated by $80 \mathrm{~nm}$ ) with different type of DW exchange interaction at $\mathrm{T}=0 \mathrm{~K}$ using the $1 \mathrm{D}$ model: (a) and (c) $\alpha=0.02$ and $\beta=0.04$, (b) and (d) $\alpha=0.05$ and $\beta=0.1$. In (a) and (b) the pulse stable time was varied, with $\mathrm{t}_{r}=\mathrm{t}_{f}=5 \mathrm{ps}$, while in (c) and (d) the raise time was varied with $\mathrm{t}_{s}=\mathrm{t}_{f}=5 \mathrm{ps}$ and $\mathrm{t}_{z}=10 \mathrm{~ns}$.

els (c) and (d) display the case of asymmetric pulse shape $\left(\mathrm{t}_{s}=\mathrm{t}_{f}=5 \mathrm{ps}\right)$. In panel $(\mathrm{a})$, the contour plots obtained with the three-type of DW exchange interaction are superposed with the results obtained with no repulsive interaction (shown in Fig. 3(a)). The influence of the different DW exchange forms is more marked for the $4 \mathrm{DWs}$, as the depinning current decreases as compared with the $2 \mathrm{DWs}$ case, to $1.54 \mathrm{~A} / \mu \mathrm{m}^{2}$ for Heisenberg DW exchange and below $1 \mathrm{~A} / \mu \mathrm{m}^{2}$ for an exponential or Yukawa -type DW exchange. In the same time, the superior bands are displaced to higher currents as compared to the 2DWs case, for example the begining of the band +1 to $4.3 \mathrm{~A} / \mu \mathrm{m}^{2}$ from $3.5 \mathrm{~A} / \mu \mathrm{m}^{2}$ (Heisenberg DW exchange). This means that higher currents are needed to achieve a synchronously movement of the DWs and a larger unintended zone. The surface of the bands is also strongly reduced when an Yukawa type interaction is used, which is the most unfavorable scenario. In the case of the asynchronous current pulse (panels (c) and (d)), the same shift of the depining current and of the bands is observed, with a clear difference between the different DW exchange schemes.

\section{B. Temperature dependence}

The effect of temperature was computed with the stochastic 1D model (Eqs. (2)) for the first bands and micromagnetically only on several points that corresponded to the highest probability obtained with the 1D model. A more detailed comparison between the analytic and micromagnetic calculated probabilities for the first band in Fig. 5(a) is shown in the Supplemental Material[53]. The results obtained analytically at $\mathrm{T}=293 \mathrm{~K}$ are presented 


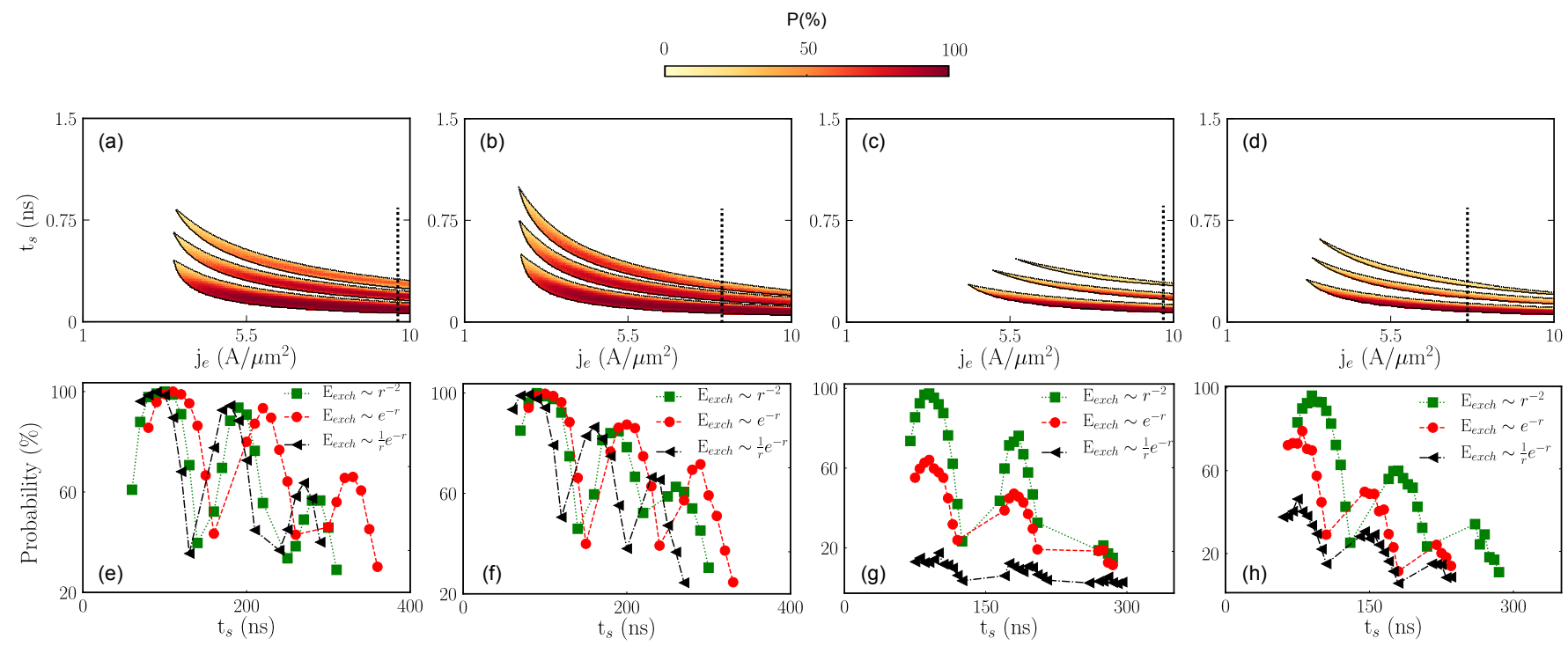

FIG. 5: (Color online) Probability of DWs motion in different bands at $\mathrm{T}=293 \mathrm{~K}$, for a train of two neighboring DWs and a damping parameter $\alpha=0.02$ in (a) or $\alpha=0.05$ in (b), or a train of four neighboring DWs with $\alpha=0.02$ in (c) or $\alpha=0.05$ in (d). A Heisenberg-type DW exchange interaction is used along with a non adiabatic parameter $\beta=2 \alpha$. The vertical dotted lines correspond to the probability profile represented in the figures underneath. The profiles are compared for different types of DW exchange interaction, and are chosen in each case as to pass through the maximum probability of the first band (the lowest branch in the figures).

in Fig. 5 for a train of two or four DWs. A symmetric current pulse $\left(\mathrm{t}_{r}=\mathrm{t}_{f}=5 \mathrm{ps}\right)$ was applied, after an initial relaxation time of $10 \mathrm{~ns}$, followed by another relaxation time of 10ns. The bands shown in panels (a) and (b) are the bands of Fig. 2(a) and (b) for Heisenberg DW exchange, while the bands displayed in panels (c) and (d) are the ones from Fig. 4(a) and (b) for Heisenberg DW exchange. We computed 1000 realizations for the +1 band and 500 realizations for the +2 and +3 bands. The realizations were calculated for half of the points in each band for the train of two DWs (panels (a) and (b)) and for all the band points for the train of four DWs (less total points in the bands).

In Fig. 5(a), the maximum of the probability distribution for the positioning of a train of two DWs to the nearest notch $(+1$ band) is of $100 \%$ obtained for 7 states (points) $(\alpha=0.02)$ out of 3093 calculated points, with $29.9 \%$ of the states having a probability superior of $95 \%$. The states that have $100 \%$ probability of desired displacement are obtained for a pulse with $\mathrm{t}_{s}=100 \mathrm{ps}$ and current amplitude superior to $9.1 \mathrm{~A} / \mu \mathrm{m}^{2}$ or $\mathrm{t}_{s}=110 \mathrm{ps}$ and $j_{e} \geq 8.5 \mathrm{~A} / \mu \mathrm{m}^{2}$. The maximum of probability decays in the superior bands, being of $95.6 \%$ on the +2 band and $68.8 \%$ on the +3 band. These probabilities are comparable with the ones when a single DW is displaced by current pulses[44]. Micromagnetically, the maximum of probability is of $98 \%$ (on 100 realizations) obtained for the same pulse characteristics that give maximum probability with the 1D model. The discrepancy is probably due to the small shift of the bands between the two models. For a damping parameter $\alpha=0.05$ (Fig. 5(b)), the maximum of the probability distribution is of $99.9 \%$ in the +1 band obtained for a lower current amplitude of $7.8 \mathrm{~A} / \mu \mathrm{m}^{2}$ and $\mathrm{at}_{s}=90 \mathrm{ps}$. The percentage of states having a probability superior to $95 \%$ is of $30.6 \%$ of the 3310 calculated states. The maximum of probability is $91 \%$ and $77 \%$ for the +2 and +3 bands.

For a train of four DWs, the maximum of the probability distribution in the +1 band decreases slowly to $97.4 \%$ ( $\alpha=0.02$, panel (c)) and $96.2 \%(\alpha=0.05$, panel (d)). The probability maximum in the +2 and +3 band is $76.8 \%$ and $28.2 \%$ respectively for $\alpha=0.02$ and $70 \%$ and $47.8 \%$ for $\alpha=0.05$ case. The current pulse characteristics for which the probability maximum is obtained are $\left(\mathrm{t}_{s}=90 \mathrm{ps}\right.$, $\left.j_{e}=9.9 \mathrm{~A} / \mu \mathrm{m}^{2}\right)$ for $\alpha=0.02$ and $\left(\mathrm{t}_{s}=90 \mathrm{ps}, j_{e}=7.8\right.$ $\left.\mathrm{A} / \mu \mathrm{m}^{2}\right)$ for $\alpha=0.05$. The probabilities when an asymmetric pulse is applied are almost equal with the ones obtained for symmetric pulses for all the cases presented above.

In the Fig. 5(e)-(h), we compare the profiles of the probability distribution when passing through the maximum of the probability in the +1 band for the different DW exchange energies considered. The profiles corresponding to $r^{-2}$ DW exchange are represented by a dotted line in the panels directly above them. There is a considerable difference in the probabilities of a train of two DWs and a train of four DWs: for the two DWs (panels (e)-(f)), the probability maximum is almost the same in the three bands for the different DW exchange interactions with only a shift of the bands along the $t_{s}$ axis. For the train of four DWs (panels (g) and (h)), the probabilities depend strongly on the spatial variation of 
the DW exchange interaction. For the $\alpha=0.02$ case, the maximum probability in the +1 band decreases to $64.1 \%$ for the exponential DW exchange and to $17.5 \%$ for the Yukawa-type interaction. For $\alpha=0.05$, the maximum probability is $79.1 \%$ for the exponential DW exchange and $46.3 \%$ for the Yukawa-type interaction. This difference can be related to the first two terms in Eq. (4), to the damping parameter through the different damping time and to the force exerted on the walls due to the interaction energy between them. The large difference in probability of the +1 state between the Heisenberg DW exchange and Yukawa DW exchange is directly imputable to the type of the repulsive energy between the DWs[53], generally the first DW depins even before the application of the current due to the large angular variation and therefore large transient effects directly related with the oscillation of the second DW (and their mutual interaction).

\section{Influence of transient effects on the DW dynamics}

Large transient effects were predicted and observed in the movement of one DW in a nanowire[37, 38, 44, 56]. These transient effect were related to the deformation of the wall in the periodic potential and produced a displacement of the wall in the direction opposite to the STT (opposite to the electron flow), corresponding to negative bands in our phase diagrams. The transient movement was determined to be proportional to the wall angle:

$$
\delta X=-\frac{\Delta}{\alpha}\left(1-\frac{\beta}{\alpha}\right) \delta \psi
$$

The transient displacement was predicted to appear for a value of the nonadiabatic parameter $\beta=0, \beta=\alpha$ and even $\beta=2 \alpha$ for a single DW submitted to ultrashort current pulses[44] comparable with the DW damping time $\tau_{d}$. In the case of interacting DWs, these transient effects still appear as is shown in Fig. 6, but they are greatly reduced (which seem to agree with a quantum-classical hybrid approach[57]). For a train of two DWs, the transient effects appear only for $\beta=0$ (or close to) when a symmetric pulse is applied (details in Ref.53) and even for $\beta=2 \alpha(\alpha=0.02)$ for an asymmetric pulse when the rise time is larger than $0.35 \mathrm{~ns}$. However, for a train of four DWs, the transient effects appear only in the case $\beta$ $=0$ and a rise time superior of $0.5 \mathrm{~ns}$ forming a reduced -1 band. These effects still appear even for a train of five DWs (Fig. 6(i)), with the -1 band shrinking rapidly.

The transient effect appear due to a combination of factors[44]: the presence of the periodic pinning potential which distorts the DWs, restoring force in the potential well, position of the DWs in the potential well at the pulse end and a low damping value. For the train of four DWs, the walls that are situated at the interior of the train are less distorted than the ones which are situated at the beginning and the end of the sequence as the interior walls fill symmetric interaction forces from both neighboring walls and are situated to the center of the potential well. The exterior walls are more deformed as they are pushed from the equilibrium position of the potential well and they escape first from the train creating unintended states.

The results obtained with the analytical model for a train of two DWs are displayed in Fig. 6(a) to (c), for the different DW exchange interactions and different initial distance between the DWs $(\alpha=0.05, \beta=0)$. When the two DWs are initially pinned in nearest neighboring notches situated $80 \mathrm{~nm}$ apart, the -1 band is obtained only for Heisenberg DW exchange and only for currents inferiors to $7.7 \mathrm{~A} / \mu \mathrm{m}^{2}$. There is a discrepancy with the micromagnetic result shown in panel $(\mathrm{d})$, where the -1 band continue to higher currents for shorter pulse length. If the two DWs are initially pinned at second neighboring notches $160 \mathrm{~nm}$ apart (panel (b)), the -1 band obtained analytically follows closely the micromagnetic one (panel (e)) even though somewhat larger. In this case the -1 band is obtained also for exponential DW exchange. Furthermore, if the two DWs are pinned initially even further away at third neighboring notches $240 \mathrm{~nm}$ apart (panel (c)), the -1 band is obtained for all three types of DW exchange interaction with almost same band width and form and very close to the micromagnetic result (panel (f)). As the two DWs are further away, the DW exchange interaction have only a limited influence and the dipolar interaction determines the form of the bands. We observe that the DW exchange interaction at shorter distances modifies the width and form of the band. For a train of three, four or five DWs, the -1 band is still obtained micromagnetically as shown in the panels (g) to (i). Analytically, we didn't obtained the -1 band for neither of the DW exchange interactions for trains of DWs superior of two and the parameters used above. This can be related to the smallness of the bands width and to the values of the DW exchange parameters, but also to the pinning potential analytical description (harmonic periodic potential). Changing the DW exchange parameters allows to obtain the negative bands but the depinning line no longer follows the micromagnetic result and differences are obtained for the others values of $\alpha$ and $\beta$. The values for the DW exchange and dipolar parameters were chosen to follow closely the depinning line and the first bands for $\beta=2 \alpha$. These parameters give semiquantitative results even for $\beta=0$ (the depinning line for example), but the limit of the 1D model is reached.

\section{DISCUSSION AND CONCLUSION}

We investigated the repulsive interaction between transverse DWs with the same topological charge, pinned at constrictions in a magnetic nanowire. Our analytical study of the DW interaction shows that a $r^{-2}$ decay de- 

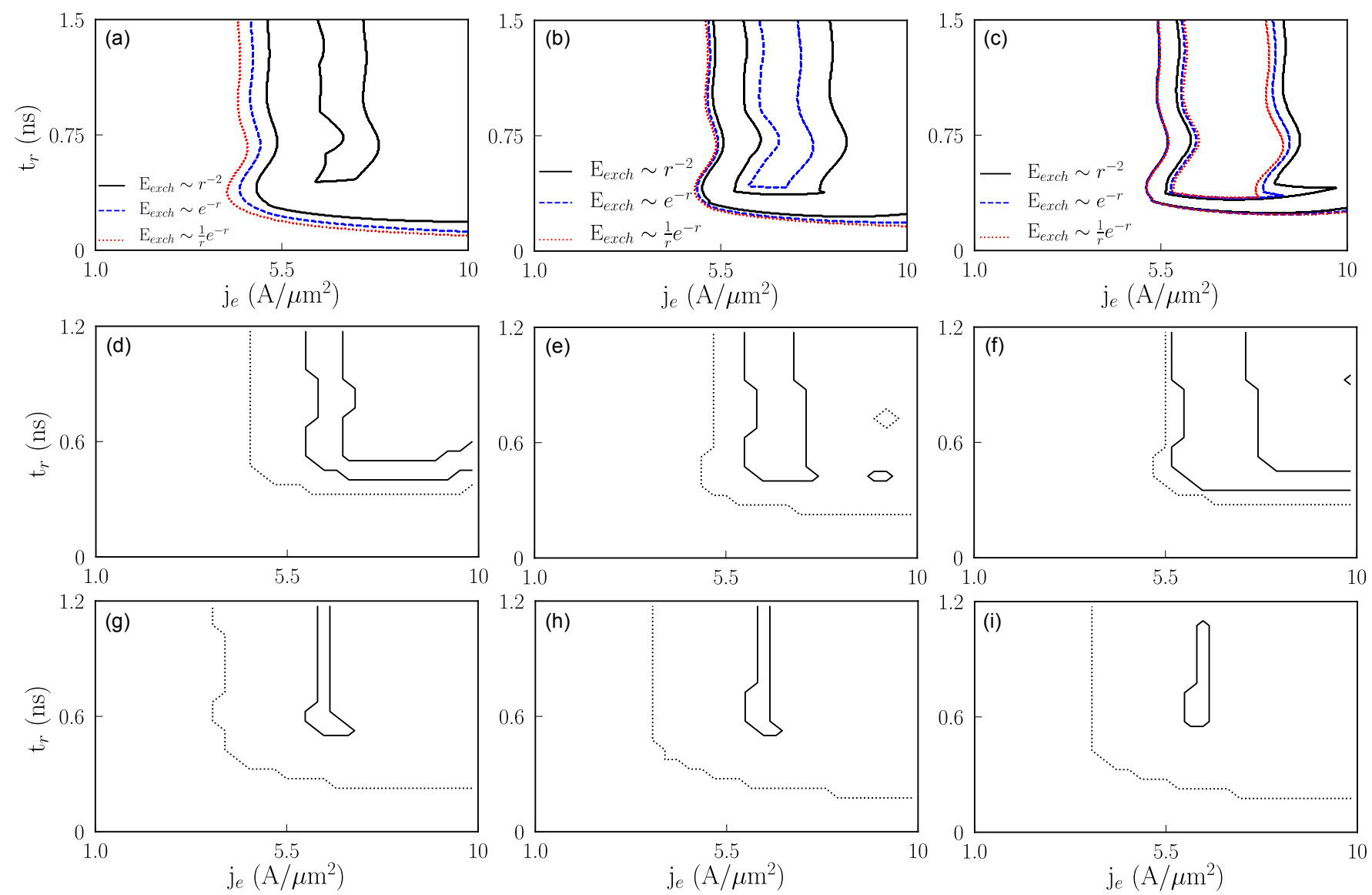

FIG. 6: (Color online) Influence of the pulse raise time $t_{r}$ on the phase diagram for a train of several DWs at $\mathrm{T}=0 \mathrm{~K}$ for $\alpha=0.05$ and $\beta=0$. The parameter space is the raise time vs. current amplitude. In all cases, $\mathrm{t}_{s}=\mathrm{t}_{f}=5 \mathrm{ps}$ and $\mathrm{t}_{z}=10 \mathrm{~ns}$. The $1 \mathrm{D}$ model results for different DW exchange energies are shown in panels (a) to (c) for a train of 2DWs separated by: (a) $80 \mathrm{~nm}$, (b) $160 \mathrm{~nm}$ and (c) $240 \mathrm{~nm}$. Only the band -1 is visible in the center of the diagram, together with the depinning boundary. The micromagnetic results are presented in the panels (d) to (i), where the band -1 is represented by a continuous line and the limit of the band 0 by the dotted line. The micromagnetic simulations are for a train of two DWs separated by: (d) $80 \mathrm{~nm}$, (e) $160 \mathrm{~nm}$ and (f) $240 \mathrm{~nm}$ and a train of (g) three DW, (h) four DW and (i) five DW separated by $80 \mathrm{~nm}$.

scribe best the micromagnetic results. The same power law variation was found to describe the vortex-vortex interaction in superconducting films[7], but differs from the vortex-vortex interaction in bulk superconductors or the magnetic vortex-vortex interaction calculated in a $2 \mathrm{D}$ anisotropic film (which decays logarithmically). Our trial functions for the interaction potential also included an exponential or Yukawa potential, which describe a large number of interaction forces in many areas of condensed matter physics (in discrete or continuum models).

The analytical phase diagrams for a train of up to four DWs follow closely the ones calculated with micromagnetic simulations, when the $r^{-2}$ decay is used. If the repulsive interaction would decay with an Yukawa potential, the changes to the phase diagrams are important starting from the depinning current and the form of the bands to the large decrease in the maximum jump probability to the nearest pinning position (the +1 band) at room temperature and the suppression of the transient effects. The Yukawa-type repulsive interaction between DWs is the most unfavorable scenario for the collective motion of DWs in a nanostrip at room temperature.

A train of four DWs is shown to be displaced regularly between pinning centers, with ultrashort current pulses (100ps), leading to practical applications like magnetic memories. The lowest depinning currents are found for the ultrashort rise time of the pulse as described before[44] as long as the largest bandwidth. When going from a train of two nearest neighboring DWs to a train of four, the main impact is the decrease of the depinning current, but in the same time a decrease of the bandwidth and an increase of the unintended region with larger transition regions between the bands. The transient effects are also severely diminished due to the mutual interaction and are eventually suppressed.

We would like to discuss our results on the repul- 
sive interaction between DWs from the spin waves perspective. The spin waves or magnons are the elementary excitations of the electronic magnetic system[58], quasiparticles with a $\hbar$ angular momentum and $\hbar k$ linear momentum. It was shown both theoretically[59-64] and experimentally[65] that the spin waves can induce DW motion in both directions due to the angular or linear momentum transfer. The motion is directly related with the transmission coefficient of the spin waves passing through the wall. It was also shown numerically, that the rigid DW motion is not stable against spin wave emission[66-69]. In principle, a DW submitted to an ultrashort current pulse could emit spin waves that interact with other DWs. This interaction will be attractive if only the angular momentum is transferred to the second DW (magnonic spin torque) or more complex if linear momentum is also transferred. The repulsive interaction considered here is thought to be mediated through the exchange of gauge bosons of integer spin which could be virtual magnons. A DW would emit a magnon that is absorbed by another DW of opposite topological charge, therefore inducing the repulsive interaction which is a fundamental interaction that exists with or without the presence of notches or an applied external current. If the DWs could rotate out-of-plane, one of the DWs could change its topological charge and the interaction can become attractive as observed in cylindrical nanowires[15]. In our view, this can be demonstrated exactly only in a microscopic theory and can not be proved in a continuum theory. In our micromagnetic simulation this interaction arises due to the exchange energy term and is described analytically like an exchange interaction between DWs (which can be described as a magnon spin current).

In summary, our calculations show that an analytical description of the interaction between several DWs is possible paving the way for larger calculations of interacting DWs in nanowires. We expect the same type of dependence to take place between Bloch or Neel DWs in thin films with perpendicular magnetic anisotropy.

\section{ACKNOWLEDGMENTS}

This work was granted access to the HPC resources of Aix-Marseille Université financed by the project Equip@Meso (ANR-10-EQPX-29-01) of the program "Investissements d'Avenir" supervised by the Agence Nationale pour la Recherche.
[1] R. Machleidt and I. Slaus, J. Phys. G: Nucl. Part. Phys. 27, R69 (2001).

[2] P. K. Shukla and K. Avinash, Phys. Rev. Lett. 107, 135002 (2011).

[3] K. Kremer, M. O. Robbins, and G. S. Grest, Phys. Rev. Lett. 57, 2694 (1986).

[4] R. Boesch, P. Stancioff, and C. R. Willis, Phys. Rev. 38, 6713 (1988).

[5] H.B.Nielsen and P.Olesen, Nucl. Phys B 61, 45 (1973).

[6] M. B. Hindmarsh and T. W. B. Kibble, Rep. Prog. Phys. $\mathbf{5 8}, 477$ (1995).

[7] A. L. Fetter and P. C. Hohenberg, Phys. Rev. 159, 330 (1967).

[8] D. Helbing, Rev. Mod. Phys. 73, 1067 (2001).

[9] W. F. Brown, Micromagnetics (Interscience Publishers, New York, London, 1963).

[10] R. D. McMichael and M.J. Donahue, IEEE Trans. Magn. 33, 4167 (1997).

[11] J. M. Kosterlitz and D. J. Thouless, J. Phys. C: Solid State Phys. 6, 1181 (1973).

[12] O. Tchernyshyov and G.-W. Chern, Phys. Rev. Lett. 95, 197204 (2005).

[13] S. K. Kim, S. Takei and Y. Tserkovnyak, Phys. Rev. B 92, 220409 (2015).

[14] S. B. Braun, Adv. Phys. 61, 1 (2012).

[15] V. O. Dolocan, Eur. Phys. J. B 87, 188 (2014).

[16] A. Pushp, T. Phung, C. Rettner, B. P. Hughes, S.-H. Yang, L. Thomas, and S. S. P. Parkin, Nat. Phys. 9, 505 (2013).

[17] S. S. P. Parkin, M. Hayashi, and L. Thomas, Science 320, 190 (2008).

[18] D. A. Allwood, G. Xiong, C. C. Faulkner, D. Atkinson,
D. Petit, R. P. Cowburn, Science 309, 1688 (2005).

[19] A. R. Völkel, F. G. Mertens, A. R. Bishop, and G. M. Wysin, Phys. Rev. B 43, 5992 (1991).

[20] A. R. Völkel, G. M. Wysin, F. G. Mertens, A. R. Bishop, and H. J. Schnitzer, Phys. Rev. B 50, 12711 (1994).

[21] K. S. Buchanan, P. E. Roy, M. Grimsditch, F. Y. Fradin, K. Yu. Guslienko, S. D. Bader, and V. Novosad, Nat. Phys. 1, 172 (2005).

[22] A. Thiaville and Y. Nakatani in Spin Dynamics in Confined Magnetic Structures III, edited by B. Hillebrands, and A. Thiaville (Springer, Berlin, 2006).

[23] M. Kläui, J. Phys.: Condens. Matter 20, 313001 (2008).

[24] J. Shibata, G. Tatara and H. Kohno, J. Phys. D: Appl. Phys. 44, 384004 (2011).

[25] T. J. Hayward, M. T. Bryan, P. W. Fry, P. M. Fundi, M. R. J. Gibbs, M.-Y. Im, P. Fischer, and D. A. Allwood, Appl. Phys. Lett. 96, 052502 (2010).

[26] T. J. Hayward, M. T. Bryan, P. W. Fry, P. M. Fundi, M. R. J. Gibbs, D. A. Allwood, M.-Y. Im, and P. Fischer, Phys. Rev. B 81, 020410 (2010).

[27] J.-S. Kim, M.-A. Mawass, A. Bisig, B. Kruger, R. M. Reeve, T. Schulz, F. Buttner, J. Yoon, C.-Y. You, M. Weigand, H. Stoll, G. Schutz, H. J.M. Swagten, B. Koopmans, S. Eisebitt and M. Klaui Nat Commun 5, 3429 (2014).

[28] A. T. Galkiewicz, L. O'Brien, P. S. Keatley, R. P. Cowburn, and P. A. Crowell, Phys. Rev. B 90, 024420 (2014).

[29] V. O. Dolocan, Appl. Phys. Lett. 105, 162401 (2014)

[30] K. Yu. Guslienko, J. Nanosci. Nanotechnol. 8, 2745 (2008).

[31] N. Hasegawa, S. Sugimoto, H. Fujimori, K. Kondou, Y. Niimi, and Y. Otani, App. Phys. Exp. 8, 063005 (2015). 
[32] L. O'Brien, A. Beguivin, D. Petit, A. Fernandez-Pacheco, D. Read and R. P. Cowburn, Phil. Trans. R. Soc. A 370, 5794 (2012).

[33] L. Thomas, M. Hayashi, R. Moriya, C. Rettner, and S.S.P. Parkin, Nat. Comm. 3, 810 (2012).

[34] B. Krüger, J. Phys.: Condens. Matter 24, 024209 (2012).

[35] A. Pivano and V. O. Dolocan, J. Magn. Magn. Mater. 393, 334 (2015).

[36] J. M. Kosterlitz, J. Phys. C: Solid State Phys. 7, 1046 (1974).

[37] A. Thiaville, Y. Nakatani, F. Piechon, J. Miltat and T. Ono, Eur. Phys. J. B 60, 15 (2007).

[38] J. Y. Chauleau, R. Weil, A. Thiaville, and J. Miltat, Phys. Rev. B 82, 214414 (2010).

[39] L. Thomas, M. Hayashi, X. Jiang, R. Moriya, C. Rettner, and S.S.P. Parkin, Nature 443, 197 (2006).

[40] L. Thomas, R. Moriya, C. Rettner, and S.S.P. Parkin, Science 330, 1810 (2010).

[41] J. Rhensius, L. Heyne, D. Backes, S. Krzyk, L. J. Heyderman, L. Joly, F. Nolting, and M. Kläui, Phys. Rev. Lett. 104, 067201 (2010).

[42] E. Martinez, L. Lopez-Diaz, O. Alejos, L. Torres, and M. Carpentieri, Phys. Rev. B 79, 094430 (2009).

[43] H. Y. Yuan and X. R. Wang, Phys. Rev. B 89, 054423 (2014).

[44] A. Pivano and V. O. Dolocan, Phys. Rev. B 96, 224431 (2017).

[45] J. C. Slonczewski, J. App. Phys. 45, 2705 (1974).

[46] A. Vansteenkiste, J. Leliaert, M. Dvornik, M. Helsen, F. Garcia-Sanchez, and B. Van Waeyenberge, AIP Advances 4, 107133 (2014).

[47] S. Zhang and Z. Li, Phys. Rev. Lett. 93, 127204 (2004).

[48] O. Boulle, G. Malinowski, and M. Kläui, Mater. Sci. Eng. R 72, 159 (2011).

[49] M. E. Lucassen, H. J. van Driel, C. Morais Smith, and R. A. Duine, Phys. Rev. B 79, 224411 (2009).

[50] H. Y. Yuan and X. R. Wang, J. Magn. Magn. Mater. 368, 70 (2014).

[51] H. Y. Yuan and X. R. Wang, Phys. Rev. B 92, 054419 (2015).

[52] K. Gilmore, Y. U. Idzerda, and M. D. Stiles, Phys. Rev. Lett. 99, 027204 (2007).

[53] See Supplemental Material at [URL will be inserted by publisher] for details of parameters influence on phase diagram

[54] B. Krüger, D. Pfannkuche, M. Bolte, G. Meier, and U. Merkt, Phys. Rev. B 75, 054421 (2007).

[55] L. Bocklage, B. Krüger, T. Matsuyama, M. Bolte, U. Merkt, D. Pfannkuche, and G. Meier, Phys. Rev. Lett. 103, 197204 (2009).

[56] J. Vogel, M. Bonfim, N. Rougemaille, O. Boulle, I. M. Miron, S. Auffret, B. Rodmacq, G. Gaudin, J. C. Cezar, F. Sirotti, and S. Pizzini, Phys. Rev. Lett. 108, 247202 (2012).

[57] M. D. Petrovic, B. S. Popescu, U. Bajpai, P. Plechac, and B. K. Nikolic, Phys. Rev. Appl. 10, 054038 (2018).

[58] A. G. Gurevich and G. A. Melkov, Magnetization Oscillations and Waves (CRC Press, Boca Raton, New York, London, 1996).

[59] D.-S. Han, S.-K. Kim, J.-Y. Lee, S. J. Hermsdoerfer, H. Schultheiss, B. Leven, and B. Hillebrands, Appl. Phys. Lett. 94, 112502 (2009).

[60] M. Jamali, H. Yang, and K.-J. Lee, Appl. Phys. Lett. 96, 242501 (2010).

[61] P. Yan, X. S. Wang and X. R. Wang, Phys. Rev. Lett. 107, 177207 (2011).

[62] J.-S. Kim, M. Stark, M. Klaui, J. Yoon, C.-Y. You, L. Lopez-Diaz and E. Martinez, Phys. Rev. B 85, 174428 (2012).

[63] X.-G. Wang, G.-H. Guo, Y.-Z. Nie, G.-F. Zhang, and Z.-X. Li, Phys. Rev. B 86, 054445 (2012).

[64] P. Yan, A. Kamra, Y. Cao, and G. E. W. Bauer, Phys. Rev. B 88, 144413 (2013).

[65] W. Jiang, P. Upadhyaya, Y. Fan, J. Zhao, M. Wang, L.T. Chang, M. Lang, K. L. Wong, M. Lewis, Y.-T. Lin, J. Tang, S. Cherepov, X. Zhou, Y. Tserkovnyak, R. N. Schwartz, and K. L. Wang, Phys. Rev. Lett. 110, 177202 (2013).

[66] D. Bouzidi, and H. Suhl, Phys. Rev. Lett. 65, 2587 (1990).

[67] J. Fernandez-Rossier, M. Braun, A. S. Nunez, and A. H. MacDonald, Phys. Rev. B 69, 174412 (2004).

[68] X.S. Wang, P. Yan, Y. H. Shen, G. E. W. Bauer, and X. R. Wang, Phys. Rev. Lett. 109, 167209 (2012).

[69] B. Hu, and X. R. Wang, Phys. Rev. Lett. 111, 027205 (2013). 


\title{
Analytical description of the topological interaction between magnetic domain walls in nanowires - Supplemental Material
}

\author{
A. Pivano and V.O. Dolocan* \\ Aix-Marseille Université, CNRS, IM2NP UMR7334, \\ F-13397 Marseille Cedex 20, France
}




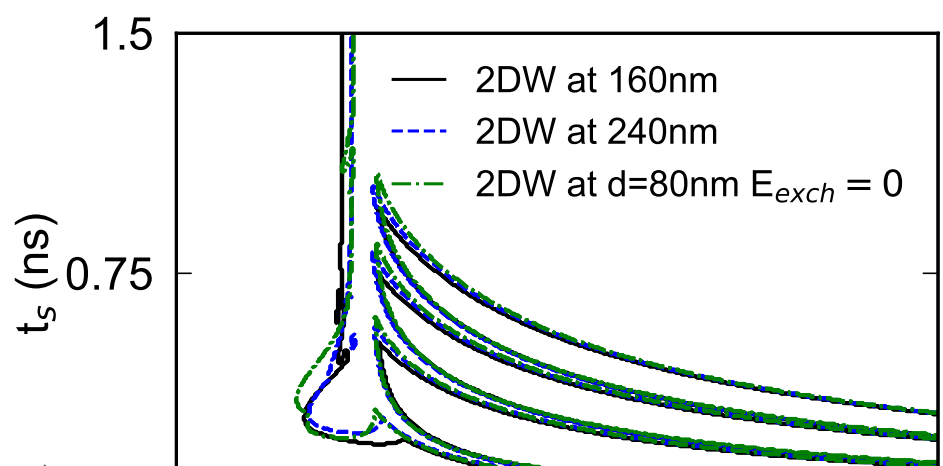

FIG. S1. (Color online) Contour plot of the first bantrained for a train of two DWs when situated at nearest neighboring:Qinning centers (80 5.5 distance) with the 10pological repulsion (DW exchange term) set to zero and when a jewer $\left(\mathrm{A} / \mathrm{am}_{r}^{2}\right)_{2} \mathrm{DW}$ interaction is considered but the DWs are situated at next-to-nearest neighbors (160 nm distance) or next-to-next-to-nearestneighbors $(240 \mathrm{~nm})$. The parameters used are: $\mathrm{t}_{r}=\mathrm{t}_{f}=5 \mathrm{ps}, \alpha=0.02, \beta=2 \alpha$.

\section{INFLUENCE OF THE DOMAIN WALLS DISTANCE ON THE PHASE DIA- GRAMS}

When two domain walls are situated at nearest neighbor pinning centers (at $80 \mathrm{~nm}$ ), the phase diagrams stable time $\mathrm{t}_{s}$ - current amplitude $\mathrm{j}_{e}$ are presented in Fig.2 of the main text for different topological interaction types. The topological interaction decays faster than the monopole-monopole interaction between the DWs, and at larger distances has lesser impact (due mainly to the demagnetizing field). This is detailed in Fig. S1, where the contour plot of the first three bands (and the depinning line) is compared for a train of two DWs situated at first neighboring pinning centers $(80 \mathrm{~nm})$ without taking into account the repulsive DW exchange interaction with the cases when the two DWs are situated at larger distances at second neighboring pinning centers and third neighboring pinning centers (160 nm and $240 \mathrm{~nm}$ respectively) with the DW exchange interaction on. The model used for the DW exchange interaction is the power law dependence $r^{-2}$ (called Heisenberg DW exchange). We see that further away, the bands become more and more comparable with the case without exchange, even though a discrepancy still exists, especially in the depinning barrier at low 


\section{COMPARISON BETWEEN THE ANALYTIC PHASE DIAGRAMS AND MI- CROMAGNETIC ONES AT $\mathrm{T}=0 \mathrm{~K}$}

The comparison between the phase diagrams obtained by analytic calculations (colors) and the ones obtained by micromagnetic simulations (symbols) are presented in Fig. S2 and Fig. S3 for a train of two neighboring DWs and a train of four neighboring DWs respectively. The phase diagrams were computed for a symmetric current pulse with $\mathrm{t}_{r}=\mathrm{t}_{f}=5$ ps and for an asymmetric current pulse with $\mathrm{t}_{s}=\mathrm{t}_{f}=5$ ps. The damping parameter $\alpha$ and the non-adiabatic parameter $\beta$ used are indicated on each figure. The analytic phase diagrams were determined using a Heisenberg $r^{-2}$ type DW exchange interaction. The micromagnetic diagrams are calculated up to $\mathrm{a}_{s}$ or $\mathrm{t}_{r}$ of $1.2 \mathrm{~ns}$ and the missing points in some cases for a pulse length inferior to $1.2 \mathrm{~ns}$ are due to a DW reaching the nanowire end at short pulse length or some special unintended states where two DWs collapse as the magnetization escapes through the third dimension ${ }^{1,2}$, the magnitude and length of the current being large. These cases appear usually at $\beta=0$ (normally above the Walker threshold), an example is shown in the video section.

Generally, a good agreement is obtained, with the exception of a few cases like the negative states at low $\alpha$ for a train of two DWs. The parameters $D_{i}$ and $a_{i}$ are given in the main text and are chosen as to follow closely the depinning line at short current pulse length. In the case of four DWs (Fig. S3), the agreement of the depinning line is very good in almost all of the cases, the only discrepancy is the state -2 on the $\alpha=0.05, \beta=0$ diagram as discussed on the main text.

\section{INFLUENCE OF THE DW EXCHANGE INTERACTION ON THE DW PO- SITION}

In Fig. S4, the difference between the Heisenberg DW exchange and Yukawa-Reid DW exchange interaction is detailed for a train of two DWs submitted at same current pulse. The current pulse corresponds to a +1 state in the micromagnetic simulations (Fig.2 of the

main text). The +1 state is obtained for a Heisenberg DW exchange (panels (a) for first 

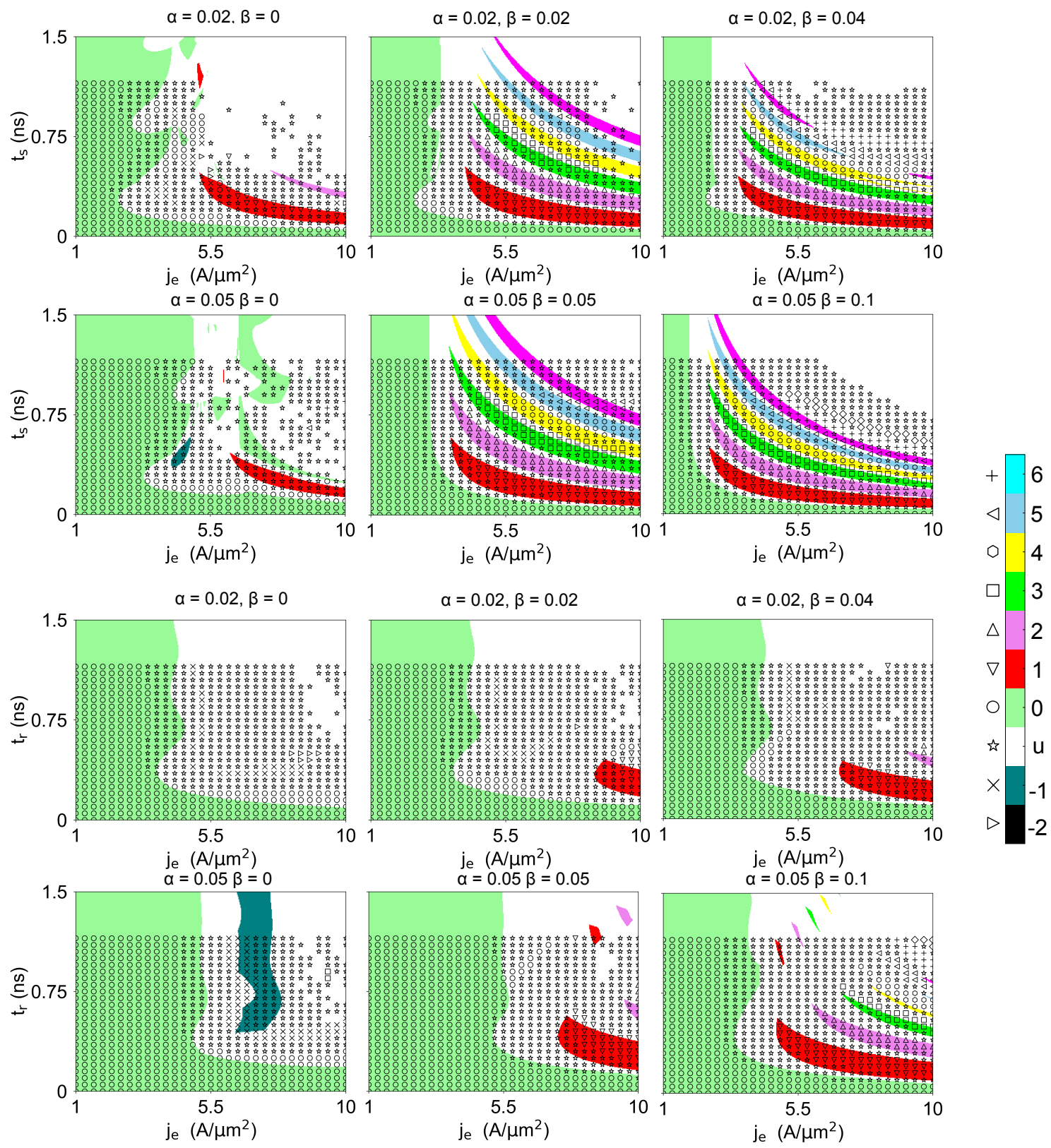

FIG. S2. (Color online) Phase diagrams of a train of two DWs situated at neighboring pinning centers, for different pulse and material parameters. The analytical calculation with the Heisenberg DW exchange interaction (colors) is superposed on the micromagnetic calculations (symbols).

DW and panel (b) for second DW), with the DW azimuthal angle oscillating around its equilibrium value (in-plane direction) for both DWs. At the end of the current pulse (the circular dot in each panel), the DW angle for both DW already oscillated once around the equilibrium position and the DWs position exceeds a little bit the equilibrium position of the pinning center, but the DWs stay in their intended positions. For a Yukawa-Reid DW 

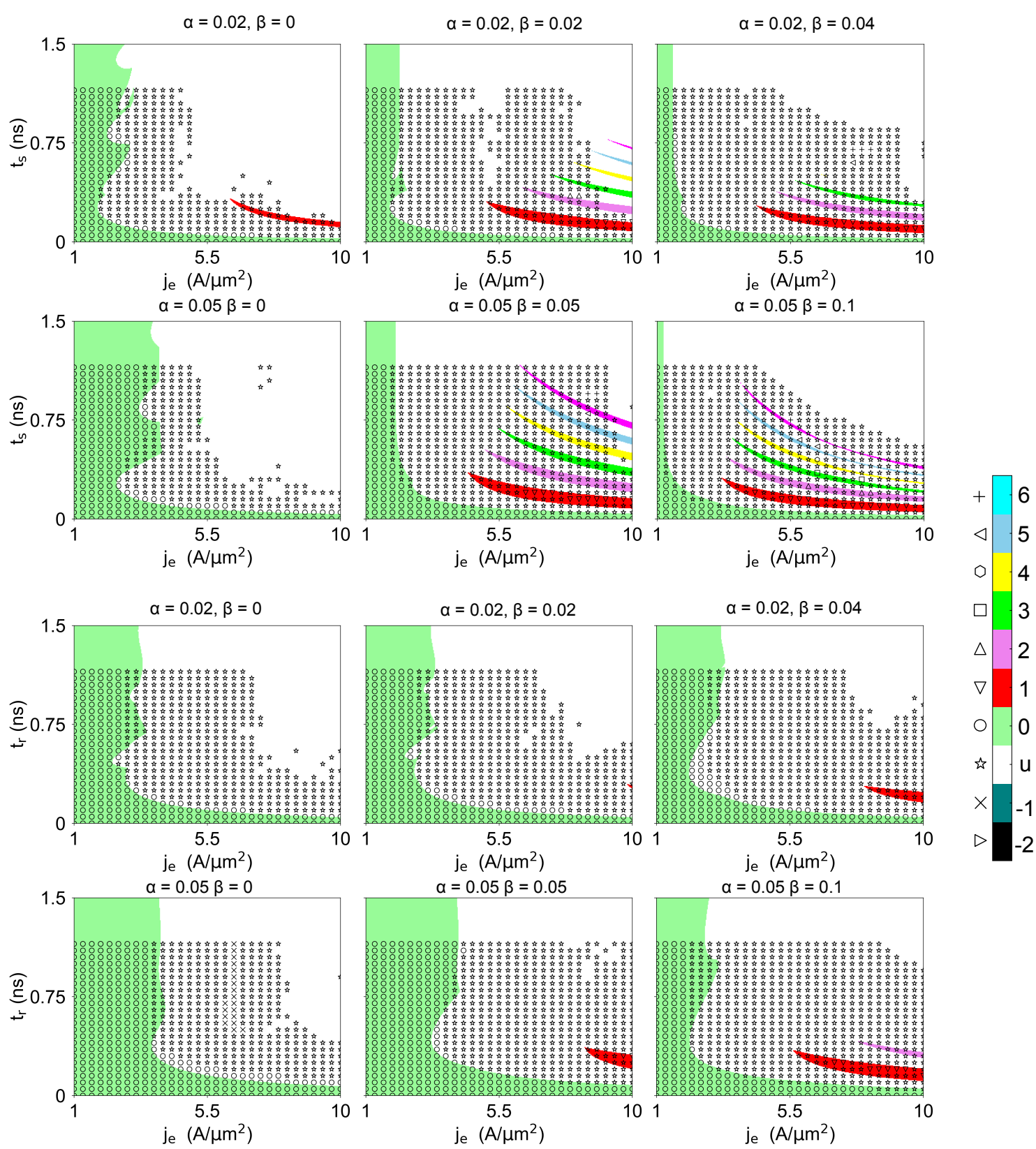

FIG. S3. (Color online) Phase diagrams of a train of four DWs situated at neighboring pinning centers, for different pulse and material parameters. The analytical calculation with the Heisenberg DW exchange interaction (colors) is superposed on the micromagnetic calculations (symbols).

exchange, the DWs angle at the end of the pulse oscillates but stays on the same side of the equilibrium position and still increases after the pulse end. The DWs position is close to the top of the potential barrier, further away from the equilibrium position compared with the Heisenberg DW exchange. The DWs have enough momentum to pass to the second neighboring position giving a +2 state after one pulse, but an unintended state after two 

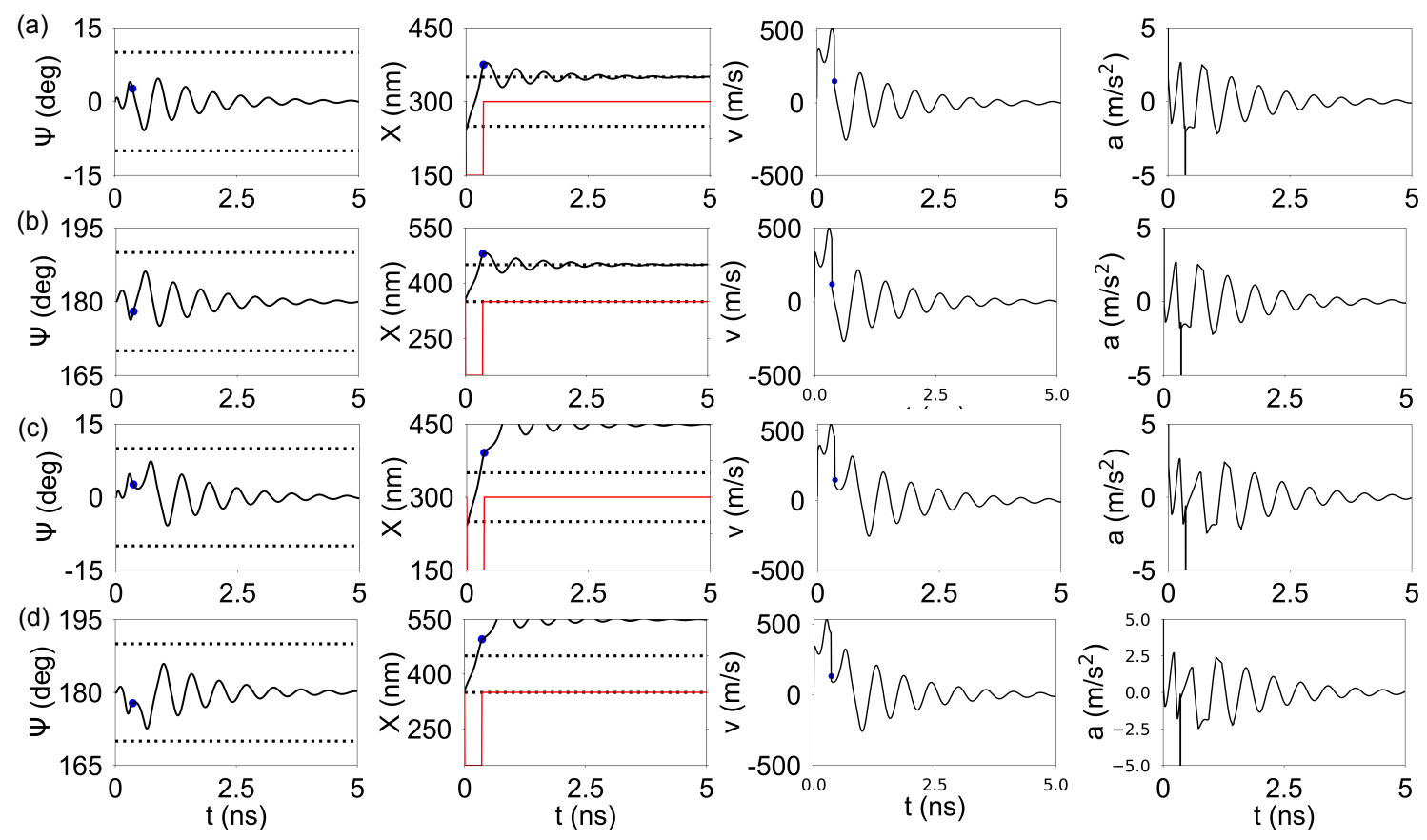

FIG. S4. (Color online) DW angular variation, position, velocity and acceleration for the first DW in (a) and (c) and second DW in (b) and (d) from a train of two neighboring DWs computed with the same pulse characteristics for a Heisenberg type DW exchange (a) and (b) and Yukawa-Reid type DW exchange in (c) and (d). The pulse parameters are: $\mathrm{t}_{r}=\mathrm{t}_{f}=5 \mathrm{ps}, \mathrm{t}_{s}=350 \mathrm{ps}$ and $\mathrm{j}_{e}$ $=-4 \mathrm{~A} / \mu \mathrm{m}^{2}$ which correspond to a +1 state with $\alpha=0.02$ and $\beta=2 \alpha$. The Yukawa-Reid DW exchange gives a +2 state after one pulse, but an unintended state after several periodic pulses. The dotted lines in the angular variation panels represent the $\pm 10^{\circ}$ around the equilibrium value, while in the position panels the dotted lines represent the position of the pinning centers in the nanowire. The current pulse shape is superimposed on the position panels. The dot represents the end of the pulse.

periodic pulses like in the main text.

\section{COMPARISON BETWEEN THE ANALYTIC PHASE DIAGRAMS AND MI- CROMAGNETIC ONES AT T=293K}

The comparison between the DWs probabilistic motion at $\mathrm{T}=293 \mathrm{~K}$, calculated with the analytic model (small dots) and by micromagnetic simulation (large dots), is shown in Fig. S5 for a train of 2 DWs and a damping parameter $\alpha=0.02(\beta=2 \alpha)$. Micromagnetically, the 


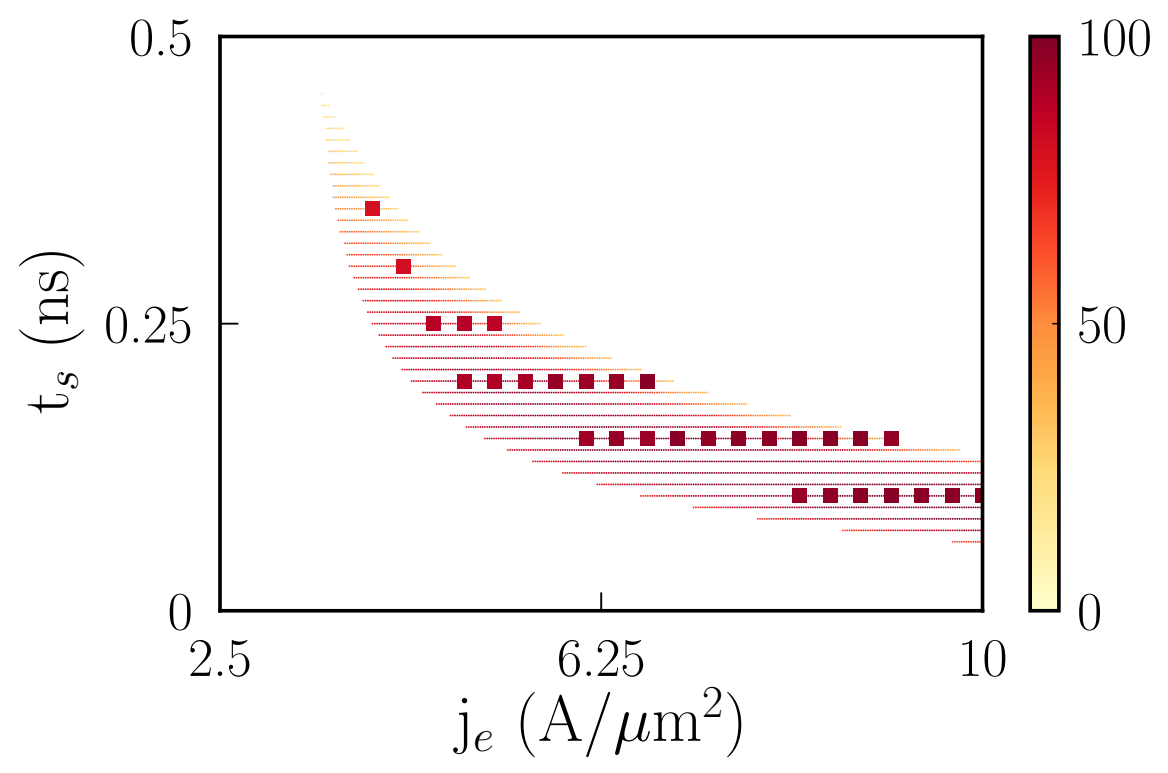

FIG. S5. (Color online) Probability of DWs motion in the +1 band for a train of two neighboring DWs at $\mathrm{T}=293 \mathrm{~K}$. The damping parameter is $\alpha=0.02$ and $\beta=2 \alpha$. The micromagnetic results on 30 band points (large squares) are superposed on the analytical results on 3093 band points (small points - almost continuous).

stochastic motion was calculated on 30 points of the +1 band, shown in the Fig.2(a) of the main article at $\mathrm{T}=0 \mathrm{~K}$, and on 100 realizations/point. The $1 \mathrm{D}$ calculated stochastic motion was on 3093 points and 1000realizations/points and was already presented in the Fig.5(a) of the main article. The maximum probability is $100 \%$ on 7 points for the $1 \mathrm{D}$ computation and $98 \%$ on 6 points for the micromagnetic one. The micromagnetic stochastic simulations are much more time consuming, therefore we only calculated the stochastic probabilities on few points for the other cases.

\section{MICROMAGNETIC SIMULATIONS - VIDEOS}

Two videos are presented with their names self-explanatory. In each video, two current pulses are applied: one at the beginning and another one after 10ns to bring the DWs at their initial position. The total simulation time is of $20 \mathrm{~ns}$. The current pulse is asymmetric with $\mathrm{t}_{s}=\mathrm{t}_{f}=5$ ps different $\mathrm{t}_{r}$. The damping parameter $\alpha=0.05$ and $\beta=0$.

- One video that presents the unintended state of a train of four DWs due to the out- 
of-plane rotation of the DW angle leading to the annihilation of two DWs. The pulse parameters are $\mathrm{t}_{r}=1.15 \mathrm{~ns}$ and $\mathrm{j}_{e}=-9.7 \mathrm{~A} / \mu \mathrm{m}^{2}$.

- One video that presents the -1 state for the same train of four DW. The pulse parameters are $\mathrm{t}_{r}=0.55 \mathrm{~ns}$ and $\mathrm{j}_{e}=-6.1 \mathrm{~A} / \mu \mathrm{m}^{2}$.

* voicu.dolocan@im2np.fr

1 H. Y. Yuan and X. R. Wang, Phys. Rev. B 92, 054419 (2015).

2 A. Pivano and V. O. Dolocan, Phys. Rev. B 96, 224431 (2017). 